\title{
Unveiling the nature of the highly absorbed X-ray source SAX J1748.2-2808 with XMM-Newton
}

\author{
L. Sidoli ${ }^{1}$, S. Mereghetti ${ }^{1}$, F. Favata ${ }^{2}$, T. Oosterbroek ${ }^{3}$, and A. N. Parmar ${ }^{2}$ \\ 1 Istituto di Astrofisica Spaziale e Fisica Cosmica - Sezione di Milano - INAF-IASF, 20133 Milano, Italy \\ e-mail: sidoli@iasf-milano.inaf.it \\ 2 Research and Scientific Support Department of ESA, ESTEC, Postbus 299, 2200 AG Noordwijk, The Netherlands \\ 3 Science Payload and Advanced Concepts Office, ESA, ESTEC, Postbus 299, 2200 AG, Noordwijk, The Netherlands
}

Received 12 April 2006 / Accepted 6 June 2006

\begin{abstract}
We report on the results of an EPIC XMM-Newton observation of the faint source SAX J1748.2-2808 and the surrounding field. This source was discovered during the BeppoSAX Galactic center survey performed in 1997-1998. A spatial analysis resulted in the detection of 31 sources within the EPIC field of view. SAX J1748.2-2808 is clearly resolved into 2 sources in EPIC images with the brighter contributing almost $80 \%$ of the $2-10 \mathrm{keV}$ flux. Spectral fits to this main source are consistent with an absorbed power-law with a photon index of $1.4 \pm 0.5$ and absorption equivalent to $14_{-4}^{+6} \times 10^{22} \mathrm{~cm}^{-2}$ together with an iron line at $6.6_{-0.2}^{+0.2} \mathrm{keV}$ with an equivalent width of $780_{-380}^{+620} \mathrm{eV}$. The significantly better statistics of the XMM-Newton observation, compared with BeppoSAX, allows to exclude a thermal nature for the X-ray emission. A comparison with other observations of SAXJ1748.2-2808 does not reveal any evidence for spectral or intensity long-term variability. Based on these properties we propose that the source is a low-luminosity high-mass $\mathrm{X}$-ray binary located in the Galactic center region.
\end{abstract}

Key words. Galaxy: center - X-rays: stars - X-rays: individual: SAX J1748.2-2808

\section{Introduction}

SAX J1748.2-2808 is an X-ray source discovered with the Narrow Field Instruments on-board BeppoSAX during a survey of the Galactic Center region (hereafter GC) performed in September 1997 (Sidoli 2000; Sidoli et al. 2001a). The BeppoSAX spectrum was severely absorbed and displayed an intense Fe K emission line. The spectrum was poorly constrained, making both thermal and non-thermal nature for the X-ray emission possible. The source, unresolved at the angular resolution of the MECS instrument $\left(F W H M \sim 1^{\prime}\right)$, is located in the direction of the giant molecular cloud Sgr D.

At the time of its discovery, the nature of SAX J1748.2-2808 was uncertain and its intense Fe $\mathrm{K}$ line emission, together with its highly absorbed spectrum, made it a unique object in the GC region which could well represent the bright tail of a distribution of similar unresolved objects significantly contributing to the diffuse Fe line emission (at $6.7 \mathrm{keV}$ ) from the galactic ridge (Koyama et al. 1989; Ebisawa et al. 2001).

Interestingly, SAX J1748.2-2808 displays properties very similar to a class of sources subsequently discovered with the INTEGRAL satellite (see e.g., Walter et al. 2004, and references therein): these objects show strong photoelectric absorption, hard 2-10 keV spectra, and often display intense Fe line emission. Most of them also show X-ray pulsations, thus indicating that they are likely high mass X-ray binaries embedded in a local absorbing gas.

Here we report the results of an XMM-Newton observation of the region of sky around SAX J1748.2-2808, performed with the main goal of unveiling the nature of this intriguing source.

\section{Observations}

The XMM-Newton Observatory (Jansen et al. 2001) includes three $1500 \mathrm{~cm}^{2} \mathrm{X}$-ray telescopes each with an European Photon Imaging Camera (EPIC) at the focus, composed of one pn (Strüder et al. 2001) and two MOS CCD detectors (Turner et al. 2001). The SAX J1748.2-2808 field was observed with XMM-Newton on 2005 February 26, 27 for about $50 \mathrm{ks}$.

Data were processed using version 6.1 of the XMM-Newton Science Analysis Software (SAS). Known hot, or flickering, pixels and electronic noise were rejected using the SAS. A further severe cleaning was necessary because of the presence of several soft proton flares. After rejecting the time intervals where the flares were present, the net good exposure times reduced to about $32.3 \mathrm{ks}$ for the MOS1 and the MOS2, and to 13.3 for the pn. Cleaned MOS1 (with pattern selection from 0 to 12), MOS2 (pattern selection from 0 to 12) and pn (patterns from 0 to 4) events files were extracted, and used for the subsequent analysis: a source detection analysis and a spectral analysis of the brightest sources.

Spectra were rebinned such that at least 20 counts per bin were present and such that the energy resolution was not oversampled by more than a factor 3 . Free relative normalizations between the MOS1, MOS2 and pn instruments were included. The background spectra were extracted from source free regions of the same observation. All spectral uncertainties and upper-limits are given at $90 \%$ confidence for one interesting parameter. 


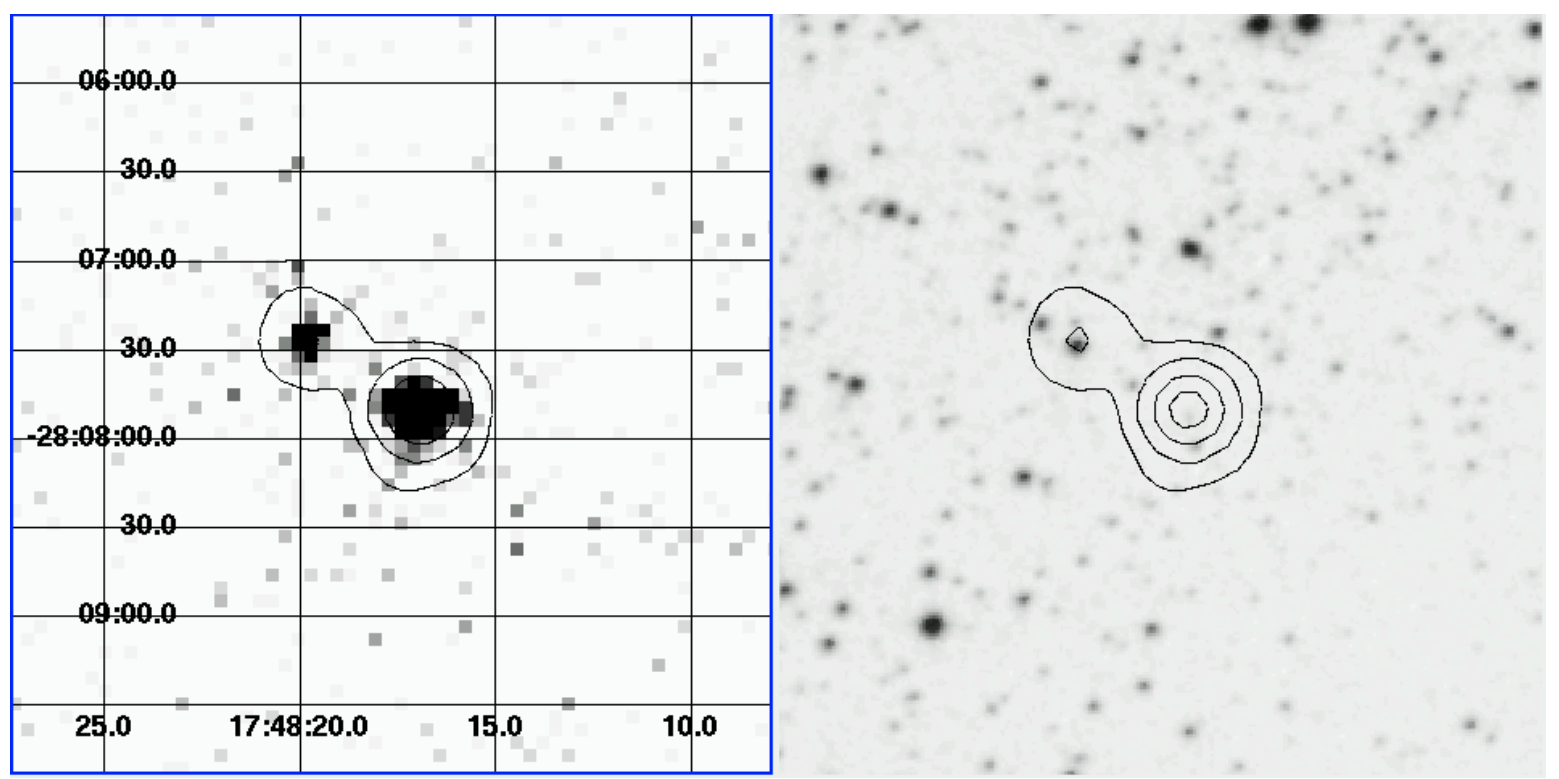

Fig. 1. Left panel: combined (pn+MOS1+MOS2) EPIC image (2-10 keV) centered on SAX J1748.2-2808. Contours (at 5, 10, 20 and 30 counts/pixel) mark the two sources (the "main" and the "faint") resolved with XMM-Newton. Right panel: optical image of the source field, from the "Second Epoch Survey" of the southern sky made by the Anglo-Australian Observatory (AAO) with the UK Schmidt Telescope (digitized plates available from STScI at http://archive.stsci.edu/). The star positionally coincident with the faint source is 0600-28834001 in the USNO-A2.0 catalog.

\section{SAX J1748.2-2808: analysis and results}

A close-up view of the combined EPIC 2-10 keV image is shown in Fig. 1, together with an optical image of the same field. XMM-Newton resolved SAX J1748.2-2808 into two sources, a brighter (here called "main" source) and a fainter one.

In order to minimize contamination from the nearby fainter source, we extracted source counts centered on the "main" source, from a circular region of $20^{\prime \prime}$ radius, for the MOS1, MOS2 and pn detectors separately.

The fit of the spectrum with an absorbed power-law $\left(\chi^{2}=\right.$ 41.5 with 35 degrees of freedom, d.o.f.) resulted in structured residuals around 6-7 $\mathrm{keV}$, confirming the presence of the $\mathrm{Fe}-\mathrm{K}$ line already observed with BeppoSAX. Adding a Gaussian line to the power-law model resulted in a significantly better fit $(\chi /$ d.o.f. $=24.9 / 32)$, with a broad line $\left(\sigma=0.43_{-0.20}^{+0.33} \mathrm{keV}\right)$ centered in the range $6.4-6.8 \mathrm{keV}$ (see Figs. 2 and 3, and Table 1 for the resulting parameters). We next tried with other simple models, such as a hot plasma model (MEKAL in XSPEC), a thermal bremsstrahlung spectrum, and a blackbody model (see Table 1 for the spectral analysis results).

The second fainter source resolved with XMM-Newton is too weak to allow a meaningful spectral analysis.

It is reasonable to assume that the SAX J1748.2-2808 emission was mostly contributed by the brighter, "main", source (and in the following we will call it SAX J1748.2-2808). The fainter source probably contributed a fraction of the measured flux from the iron line in the BeppoSAX spectrum. In order to allow a proper comparison with the BeppoSAX observation, we extracted a combined XMM-Newton spectrum from both the main and the secondary fainter source. The residuals to the fit with an absorbed power-law again clearly show an excess around 6.5-6.7 keV (see Fig. 4), requiring the addition of a Gaussian emission line. The line centroid is $6.6_{-0.1}^{+0.2} \mathrm{keV}$, the normalization is $\left(8_{-3}^{+9}\right) \times 10^{-6}$ photons $\mathrm{cm}^{-2} \mathrm{~s}^{-1}$ and the equivalent width is $400_{-150}^{+500} \mathrm{eV}$. The absorbing column density is $\left(13_{-3}^{+7}\right) \times 10^{22} \mathrm{~cm}^{-2}$, while the photon index, $\Gamma$, is $1.2_{-0.3}^{+0.9}$ (which is harder than the

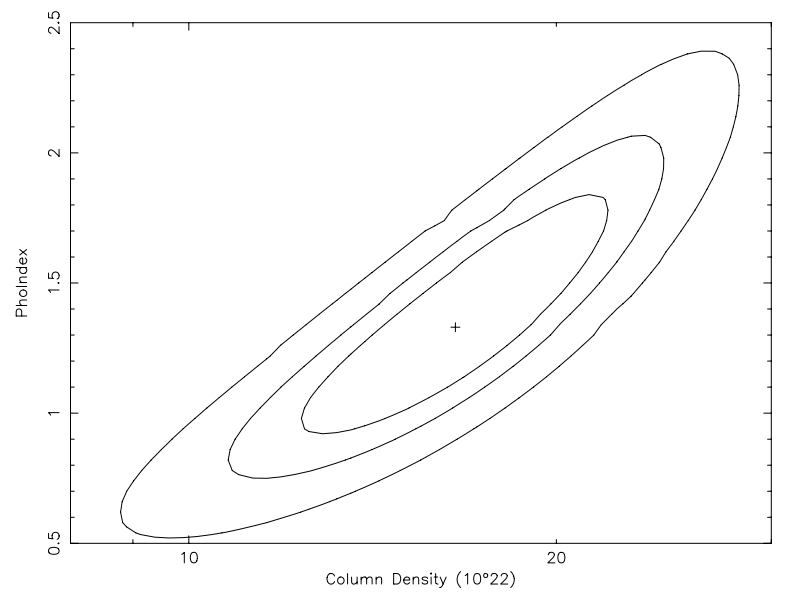

Fig. 2. 68\%, 90\% and 99\% confidence level contours for the two quantities derived with the absorbed power-law fit to the "main" source spectrum, absorbing column density and power-law photon index.

power-law fit to the "main" source alone, likely due to the hard emission contributed by the fainter source). The observed flux is $9 \times 10^{-13} \mathrm{erg} \mathrm{cm}^{-2} \mathrm{~s}^{-1}(2-10 \mathrm{keV})$. We then re-extracted the BeppoSAX MECS spectrum, and deconvolved it with this best fit model for the XMM-Newton main plus faint sources emission. The result is shown in Fig. 5 and demonstrates that there is no evidence for dramatic changes in the flux level and spectral shape.

\section{A source catalog of the SAX J1748.2-2808 field}

Examination of the EPIC images of the SAX J1748.2-2808 field in different energy ranges shows a region rich in faint $\mathrm{X}$-ray sources. We performed a detection analysis in order to obtain a source catalog of this region, using the source detection procedure described in Baldi et al. (2002). All the source detection chain uses SAS version 6.1 tasks. 

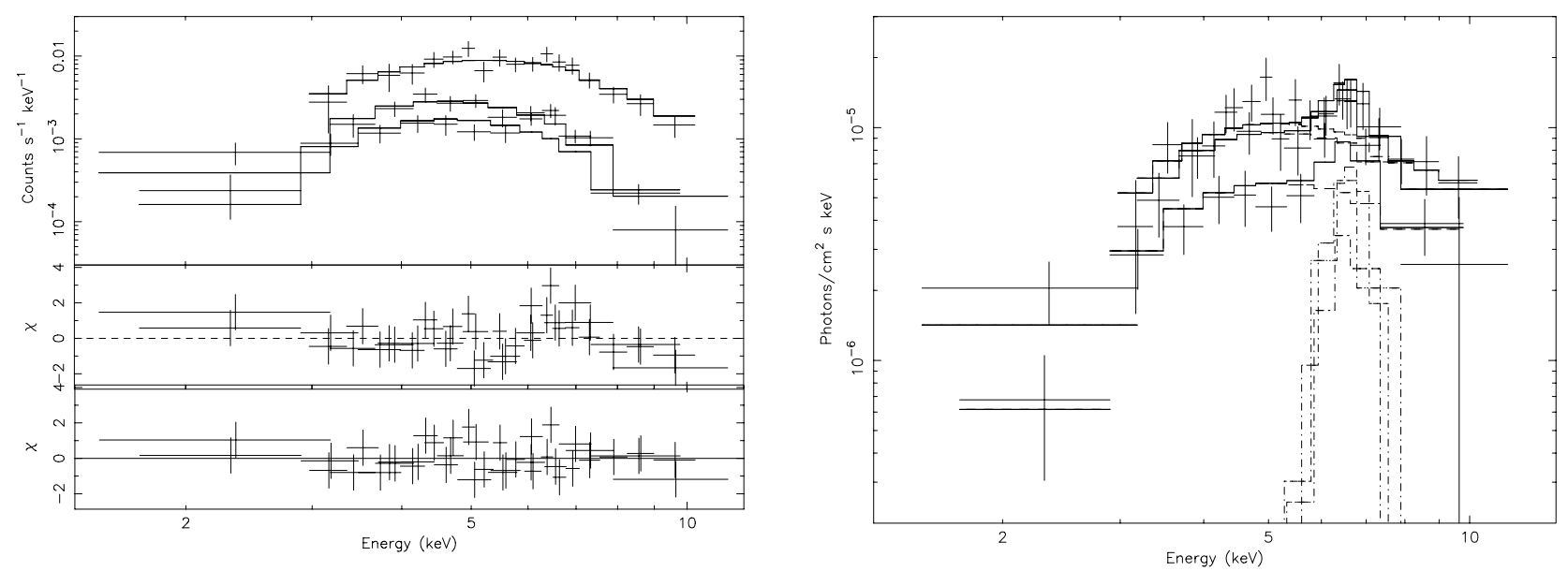

Fig. 3. Left panel: fit to the "main" source spectrum (MOS1, MOS2 and pn data) with a single absorbed power-law (top panel) together with its residuals in units of standard deviations (middle panel). There is evidence for an excess around 6-7 keV, indicative of the presence of an emission line. The residuals after adding a Gaussian line to the power-law model are shown in the bottom panel, in units of standard deviations. Right panel: photon spectrum resulting from the best-fit, composed by a power-law together with an emission line at $6.6 \mathrm{keV}$ (see Table 1 for the parameters).

Table 1. Results obtained fitting the "main" source spectrum. The $2-10 \mathrm{keV}$ flux has not been corrected for the interstellar absorption. The luminosity, $L$, has been obtained from the unabsorbed flux, assuming the GC distance $(8.5 \mathrm{kpc})$. $E_{\text {line }}$ is the Gaussian line centroid in keV, and $E W$ is the associated equivalent width $(\mathrm{keV}), \sigma$ is the line width in $\mathrm{keV}$, while $I_{\text {line }}$ is the total $10^{-6}$ photons $\mathrm{cm}^{-2} \mathrm{~s}^{-1}$ in the line.

\begin{tabular}{llllll}
\hline \hline Model & $\begin{array}{l}\text { Column density } \\
\left(10^{22} \mathrm{~cm}^{-2}\right)\end{array}$ & Parameter & $\chi^{2} /$ d.o.f. & $\begin{array}{l}\text { Flux }(2-10 \mathrm{keV}) \\
\left(10^{-13} \mathrm{erg} \mathrm{cm}^{-2} \mathrm{~s}^{-1}\right)\end{array}$ & $\begin{array}{l}L(2-10 \mathrm{keV}) \\
\left(10^{34} \mathrm{erg} \mathrm{s}^{-1}\right)\end{array}$ \\
\hline Power law & $16.5_{-4.5}^{+6.0}$ & $\Gamma=1.3_{-0.3}^{+0.6}$ & $41.5 / 35$ & 6.8 & 1.1 \\
Power law + line & $14.3_{-4.0}^{+6.0}$ & $\Gamma=1.4_{-0.5}^{+0.4}$ & $24.9 / 32$ & 6.6 & \\
& & $E_{\text {line }}=6.6_{-0.2}^{+0.2}$ & & & \\
& & $\sigma=0.43_{-0.20}^{+0.33}$ & & & \\
& & $E W=0.78_{-0.38}^{+0.62}$ & & & 1.1 \\
& & $I_{\text {line }}=9.0_{-4.1}^{+7.0}$ & & \\
MEKAL & $16.6_{-2.7}^{+2.7}$ & $T_{\mathrm{M}}=30_{-15}^{+50} \mathrm{keV}$ & $38.1 / 35$ & 6.7 & 1.1 \\
Bremsstrahlung & $16.8_{-3.2}^{+4.8}$ & $T_{\mathrm{br}}>12 \mathrm{keV}$ & $41.3 / 35$ & 6.8 & 0.8 \\
Black body & $10.4_{-3.2}^{+3.9}$ & $T_{\mathrm{bb}}=2.2_{-0.3}^{+0.4} \mathrm{keV}$ & $37.5 / 35$ & 6.7 & \\
& & $R_{\mathrm{bb}}=0.07_{-0.02}^{+0.02} \mathrm{~km}$ & & & \\
\hline
\end{tabular}

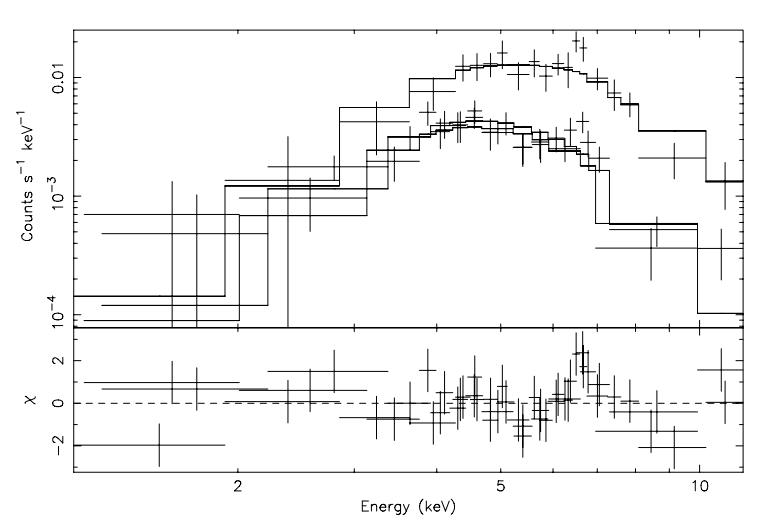

Fig. 4. XMM-Newton counts spectrum, of the "main" plus "faint" sources, fitted using an absorbed power-law (see text for the parameters), together with the residuals in units of standard deviations.

The cleaned events files were used to produce MOS1, MOS2 and pn images and exposure maps (which also include the vignetting effect) in 4 energy ranges: $0.5-2 \mathrm{keV}$ (soft band, hereafter "S"), 2-5 keV (medium band, " $M$ "), 5-10 keV (hard band, " $H$ "), and $0.5-10 \mathrm{keV}$ (total band). For each energy band independently, the MOS1, MOS2 and pn images (and the corresponding instrumental exposure maps) were then merged in

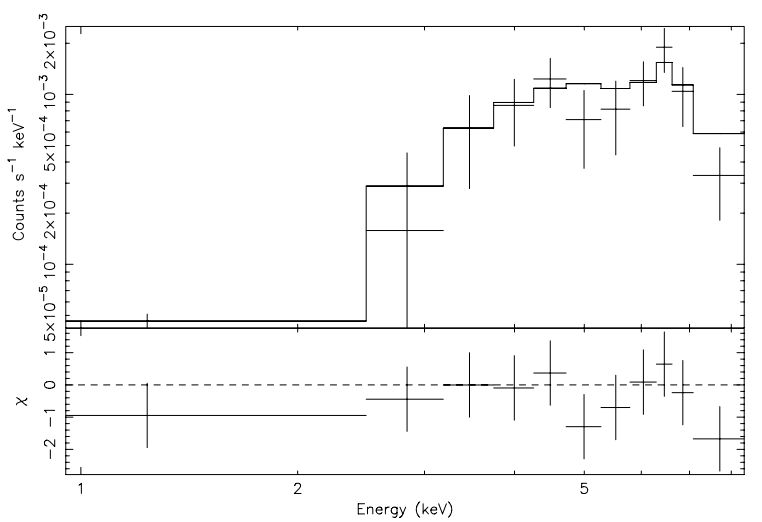

Fig. 5. BeppoSAX MECS2+MECS3 spectrum (data points) comprising the emission of both the "main" and "faint" sources, unresolved by this instrument. The line is the XMM-Newton best fit model to the sum of the two sources (Fig. 4) folded through the BeppoSAX response.

order to get a higher signal-to-noise ratio in the source detection. After the production of a detector mask (with the task emask), a source detection in local mode was performed in each energy band separately with the task eboxdetect to produce a preliminary list of sources using a sliding box technique. Then, with task esplinemap, all the sources in the list were removed from 
Table 2. X-ray spectral results for the brightest sources detected in the SAX J1748.2-2808 field (the source ID is the same as in Table 4). The flux has not been corrected for interstellar absorption and is in units of $10^{-13} \mathrm{erg} \mathrm{cm}^{-2} \mathrm{~s}^{-1}$ in the energy range $2-10 \mathrm{keV}$, except for source 6 , which is in the $0.5-2 \mathrm{keV}$ range. Al the source spectra have been fit with a power-law, except for source 6 , where a blackbody has been used (the blackbody temperature, $T_{\mathrm{bb}}$, is in $\mathrm{keV}$ ).

\begin{tabular}{llll}
\hline \hline Src ID & $\begin{array}{l}N_{\mathrm{H}} \\
\left(10^{22} \mathrm{~cm}^{-2}\right)\end{array}$ & Parameter & $\begin{array}{l}\text { Flux } \\
(2-10 \mathrm{keV})\end{array}$ \\
\hline 1 & $1.3_{-0.3}^{+0.3}$ & $\Gamma=1.7_{-0.1}^{+0.2}$ & 5 \\
2 & $8_{-2}^{+2}$ & $\Gamma=2.0_{-0.6}^{+0.4}$ & 20 \\
4 & $15_{-6}^{+5}$ & $\Gamma=1.6_{-0.9}^{+0.9}$ & 30 \\
5 & $0.7_{-0.4}^{+0.8}$ & $\Gamma=1.8_{-0.6}^{+0.8}$ & 2 \\
6 & $<0.2$ & $\mathrm{~T}_{\mathrm{bb}}=0.2 \pm 0.1$ & $0.5(0.5-2 \mathrm{keV})$ \\
7 & $1.6_{-0.7}^{+1.3}$ & $\Gamma=2.1_{-0.6}^{+1.2}$ & 1.4 \\
\hline
\end{tabular}

the image and the resulting source-free image was fitted with a cubic spline function in order to create a background map for each energy band. Then, a second run with eboxdetect in map mode was made, this time using the background maps produced before. Lastly, the final source positions, together with the EPIC combined (MOS1+MOS2+pn) count rates for each source in each energy range, were calculated using the task emldetect, which performs maximum likelihood fits to the source spatial count distribution in all energy bands. This detection procedure resulted in 31 sources, reported in Table 4. Most of them have been detected in the medium and hard energy ranges, while few sources were detected only in the soft band $(0.5-2 \mathrm{keV})$, very likely foreground stars.

The observed fluxes have been calculated from the combined EPIC count rate, $c r$, and a total conversion factor $f($ flux $=f \cdot c r)$ derived as in Baldi et al. (2002):

$\frac{T_{\mathrm{tot}}}{f}=\frac{T_{\mathrm{MOS} 1}}{f_{\mathrm{MOS} 1}}+\frac{T_{\mathrm{MOS} 2}}{f_{\mathrm{MOS} 2}}+\frac{T_{\mathrm{pn}}}{f_{\mathrm{pn}}}$,

where $T_{\text {tot }}$ is the sum of the exposure times from the three instruments, $T_{\text {tot }}=\left(T_{\mathrm{MOS} 1}+T_{\mathrm{MOS} 2}+T_{\mathrm{pn}}\right)$, and $f_{\mathrm{MOS} 1}, f_{\mathrm{MOS} 2}$ and $f_{\mathrm{pn}}$ are the single count-rate-to-flux conversion factors.

The sources listed in our catalog show a distribution of hardness ratios, ranging from sources detected only in the soft band (likely foreground stars), up to extremely hard sources, detected only above $5 \mathrm{keV}$ (see Fig. 6). In order to account for the different source hardness, we derived three different total conversion factors $f$, assuming three kinds of "typical" spectra: for sources detected only in the "S" band, we considered a blackbody spectrum with temperature $200 \mathrm{eV}$ and a column density of $10^{21} \mathrm{~cm}^{-2}$ (and the derived flux is limited to the $0.5-2 \mathrm{keV}$ energy range); for sources detected only in the " $H$ " band, we have chosen a power-law spectrum with a photon index $\Gamma=2$ and an absorbing column density of $10^{24} \mathrm{~cm}^{-2}$ (flux derived in the 5-10 keV energy range); a power-law spectrum with a photon index $\Gamma=2$ absorbed with $N_{\mathrm{H}}=2 \times 10^{23} \mathrm{~cm}^{-2}$ has been considered for all other sources (flux derived in the $2-10 \mathrm{keV}$ range).

The absorbed fluxes calculated in this way are reported in Table 4 for the faint sources, while for the brightest ones, for which a reliable spectrum can be obtained, the flux measured from the spectral analysis is reported.

A search in the SIMBAD database resulted in two HD stars positionally coincident with two soft X-ray sources (see Table 4): HD 316290, located 3"'1 from source 11, and HD 161824, at $1^{\prime \prime}$ from the soft X-ray source 15 . A third star,
Tyc2 929, is located within 1'.2 from the soft X-ray source 28. In Table 3 we report the sky positions and $B, V$ magnitudes of these 3 optical counterparts, together with the associated ratios between X-ray and optical flux (Maccacaro et al. 1988), which strengthen the physical association with the soft X-ray sources.

In the search for counterparts of the X-ray sources at other wavelengths, we have conservatively assumed a circular uncertainty region with a radius of $4^{\prime \prime}$. The results from a search in the SIMBAD database are reported in Table 4 (last column) while a cross-correlation with the 2MASS All-Sky Catalog of Point Sources (Cutri et al. 2003) resulted into 68\% X-ray matches with 2MASS counterparts.

Within the $14^{\prime}$ radius of the X-ray field of view, the $2 \mathrm{MASS}$ catalog (Cutri et al. 2003) list 26413 stars, translating into a surface density $\mu \sim 1.19 \times 10^{-2}$ sources $\operatorname{arcsec}^{-2}$. This corresponds to 0.6 sources within each error region of $4^{\prime \prime}$ radius. Therefore it is likely that a large number of the 2MASS counterparts are just random coincidences, as suggested also by the fact that several $\mathrm{X}$-ray sources are positionally associated with more than one infrared counterpart. At the spatial resolution of XMM-Newton, stellar confusion in the Galactic plane prevents from unambiguously associating infrared sources with the X-ray ones. Thus, we will not discuss further the possible association with 2MASS sources. On the other hand, for the two brightest HD stars, we estimate a probability of chance coincidence around $0.02 \%$. This, together with the measured $\log \left(f_{\mathrm{X}} / f_{V}\right)$, confirm the real association of the brightest stars with the X-ray sources.

Among the brightest X-ray sources, for which a spectral analysis has been possible, few can be firmly identified with known objects. Source 2 in Table 4 is a transient discovered with EPIC in an XMM-Newton observation performed on 12 March 2003, pointed on the composite SNR G0.9+0.1 (Sidoli $\&$ Mereghetti 2003; Sidoli et al. 2004). The observed flux during the discovery observation was $3.7 \times 10^{-12} \mathrm{erg} \mathrm{cm}^{-2} \mathrm{~s}^{-1}$ $(2-10 \mathrm{keV})$, almost a factor of 2 higher than that measured in February 2005, while the spectral parameters remained constant, within the uncertainties. The detection of the transient in 2005 could indicate that we are observing a second outburst from the source, or that the source is still in outburst since 2003. Source 4 is the pulsar wind nebula in the supernova remnant G0.9+0.1 (Mereghetti et al. 1998). The spectral parameters are similar to those measured during previous observations with XMM-Newton (Porquet et al. 2003; Sidoli et al. 2004). Source 6 is positionally coincident with source 80 in the ROSAT catalog of the GC sources (Sidoli et al. 2001b). Compared with the ROSAT observation, it displays variability.

Five faint X-ray sources have been detected only above $5 \mathrm{keV}$. While it is somehow expected that the faintest sources be more distant and absorbed, on the other hand it is remarkable that they are observed only above $5 \mathrm{keV}$, meaning that their spectrum is both truly hard and absorbed. Few of these faint hard sources could be background AGNs. From the $\log (N)-$ $\log (S)$ measured in the energy range 5-10 keV (Baldi et al. 2002) with XMM-Newton, the expected number of hard sources with flux larger than $5 \times 10^{-14} \mathrm{erg} \mathrm{cm}^{-2} \mathrm{~s}^{-1}$ is 5-11 sources $\mathrm{deg}^{-2}$, which translates into 1-2 extragalactic hard X-ray sources in the XMM-Newton field of view. The remaining sources are probably CVs located close to the GC distance (their fluxes translate into luminosities in the range $\sim 10^{32}-10^{33} \mathrm{erg} \mathrm{s}^{-1}$ ).

\section{Discussion and conclusions}

In Sidoli et al. (2001a) we reported the discovery of a new X-ray source in the direction of the Sgr D region, SAX J1748.2-2808. 

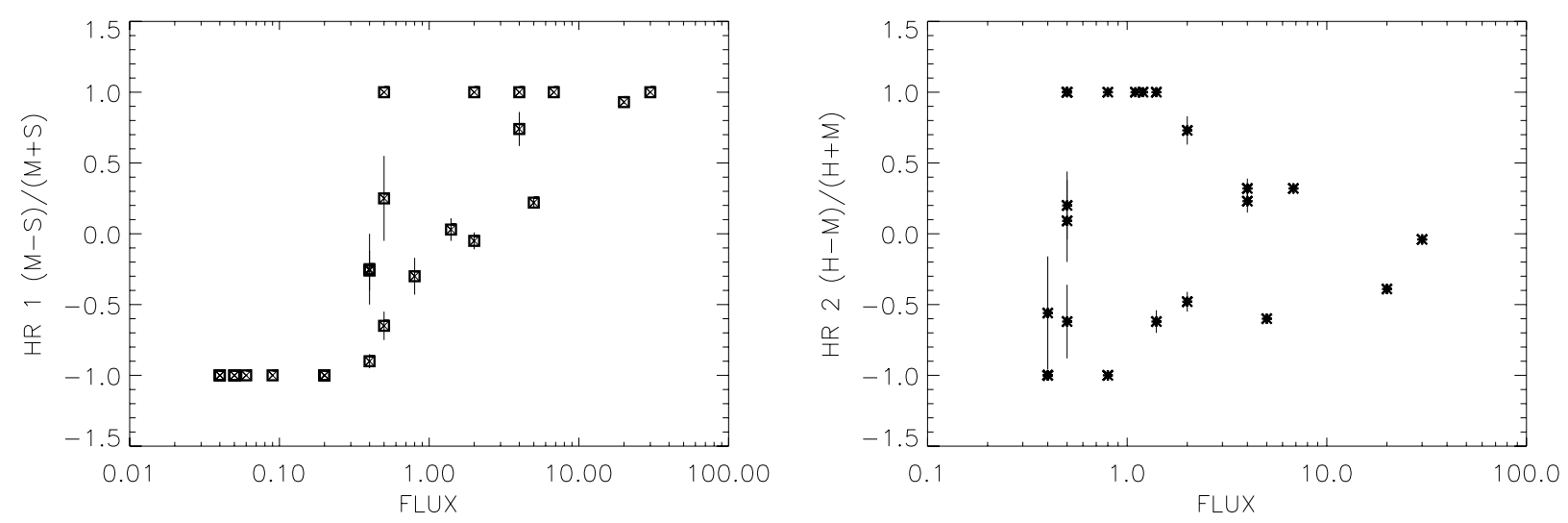

Fig. 6. Hardness ratio versus estimated flux in the energy range $2-10 \mathrm{keV}$ for the X-ray sources in the SAX J1748.2-2808 field. On the left the hardness ratio $H R 1((M-S) /(M+S))$ versus flux is shown, while in the right panel the distribution of the hardness ratio $H R 2((H-M) /(H+M))$ versus observed flux is reported. Fluxes are in units of $10^{-13} \mathrm{erg} \mathrm{cm}^{-2} \mathrm{~s}^{-1}$ (see Table 4).

Table 3. Stars positionally coincident with 3 soft X-ray sources in our catalogue.

\begin{tabular}{llllllll}
\hline \hline Star & RA (J2000) & Dec (J2000) & $\begin{array}{l}B \\
(\mathrm{mag})\end{array}$ & $\begin{array}{l}V \\
(\mathrm{mag})\end{array}$ & Sp. type & $\begin{array}{l}\text { Associated } \\
\text { X-ray source ID }\end{array}$ & $\log \left(f_{\mathrm{X}} / f_{V}\right)$ \\
\hline HD 316290 & 267.203000 & -28.016972 & 10.25 & 9.76 & F8 & 11 & -4.3 \\
HD 161824 & 267.212250 & -28.246139 & 9.63 & 8.33 & K1/K2III & 15 & -4.5 \\
Tyc2 929 & 267.045025 & -28.308665 & 12.27 & 10.55 & & 28 & -4.8 \\
\hline
\end{tabular}

Our new XMM-Newton observation allows to resolve it into two sources (sources 3 and 12 in Table 4), with a brighter "main" source contributing almost $80 \%$ of the source flux in the $2-$ $10 \mathrm{keV}$ energy range.

The fainter source is harder (detected only above $5 \mathrm{keV}$ ) than the "main" one. A possible optical counterpart is the star 0600-28834001 of the USNO-A2.0 catalog $(B=18.1, R=$ 13.4), which is listed as [RHI84]10-672 in the Raharto et al. (1984) catalog of M-type stars. The derivation of $\log \left(f_{\mathrm{X}} / f_{V}\right)$ is highly uncertain, but assuming, e.g., a blackbody emission at $k T \sim 1 \mathrm{keV}$, absorbed with a column density of $10^{24} \mathrm{~cm}^{-2}$, the 5-10 keV flux translates into a $0.3-3.5 \mathrm{keV}$ flux $\sim 4 \times$ $10^{-11} \mathrm{erg} \mathrm{cm}^{-2} \mathrm{~s}^{-1}$ (corrected for the absorption), and to a $\log \left(f_{\mathrm{X}} / f_{V}\right) \sim 1.8$, clearly not stellar. Thus, the hardness of the $\mathrm{X}$ ray emission excludes a coronal emission for the fainter source.

The refined sky position of the brighter source allows to reject all the possible associations discussed in Sidoli et al. (2001a). The BeppoSAX spectrum was affected by a high interstellar absorption, $N_{\mathrm{H}} \sim 10^{23} \mathrm{~cm}^{-2}$, suggesting that the source is probably located at the GC distance (in this case the luminosity in the $2-10 \mathrm{keV}$ energy band is $\sim 10^{34} \mathrm{erg} \mathrm{s}^{-1}$ ). A strong Fe K line was present (with a line centroid of $6.62 \pm 0.30 \mathrm{keV}$ ), and a good fit was obtained both with a power-law plus a Gaussian line, and with a hot thermal plasma model with a temperature, $k T$, of $6_{-4}^{+35} \mathrm{keV}$. Thus, the BeppoSAX spectrum was consistent with both thermal and non-thermal models.

The significantly better statistics of the XMM-Newton spectrum and the smaller uncertainties in the spectral slope, favor a non-thermal nature for the X-ray emission of the "main" source. A hard power-law $(\Gamma \sim 1.4)$ is a good fit to the data, with an iron line and a high photoelectric absorption $\left(N_{\mathrm{H}}=10-20 \times 10^{22} \mathrm{~cm}^{-2}\right)$. The absorption is probably not intrinsic, since the source is located within about 1 degree from the direction of the Galactic center. Thermal models do not fit the X-ray spectrum as well, and result in very high temperatures (for example, a thermal plasma should be hotter than $15 \mathrm{keV}$ ). Among the thermal models tried, the blackbody is the best in fitting the spectrum, but results in a high temperature $(\sim 2 \mathrm{keV})$ and in an emitting region of less then $0.1 \mathrm{~km}$ at the galactic center distance. Thus, the X-ray spectral shape favors a non-thermal nature for the X-ray emission.

The X-ray emission appears to be stable; the "main" source has been detected at large off-axis angle during two previous XMM-Newton observations performed in September 2000 and March 2003 (both pointed at the SNR G0.9+0.1). In both occasions, SAX J1748.2-2808 did not show evidence for any strong flux variability. Moreover, the total emission from "main" plus "faint" source, is compatible with that observed with BeppoSAX in 1997 (see Fig. 5).

These properties are suggestive of three possibilities: a binary system containing a compact object, a background AGN, or reflection from a molecular cloud core (e.g., similar to the $\mathrm{X}$-rays emission and fluorescent iron line produced from the molecular cloud Sgr B2; Revnivtsev et al. 2004). This third possibility, already discussed in Sidoli et al. (2001a), seems now unlikely, based on the high spatial resolution of the XMM-Newton observation. The compact cores contained in the giant molecular cloud Sgr B2, for example, are about 1 pc in size (Lis \& Goldsmith 1991), while the XMM-Newton spatial resolution $\left(F W H M \sim 6^{\prime \prime}\right)$ allows us to exclude a source with a size larger than $\sim 0.25 \mathrm{pc}$ at a distance of $8.5 \mathrm{kpc}$.

The shape of the X-ray spectrum and the parameters of the iron line are consistent with a background AGN. It should be a nearby object, since the Fe line is not red-shifted. Assuming a distance of $5 \mathrm{Mpc}$, the $2-10 \mathrm{keV}$ unabsorbed flux corresponds to a luminosity of $\sim 3.4 \times 10^{39} \mathrm{erg} \mathrm{s}^{-1}$, which is quite low, but still compatible with a low-luminosity Seyfert galaxy (Terashima et al. 2002). Note that no radio counterpart is present in the NED catalogue within $30^{\prime \prime}$ of the X-ray position, and SAX J1748.2-2808 does not show evidence for X-ray 


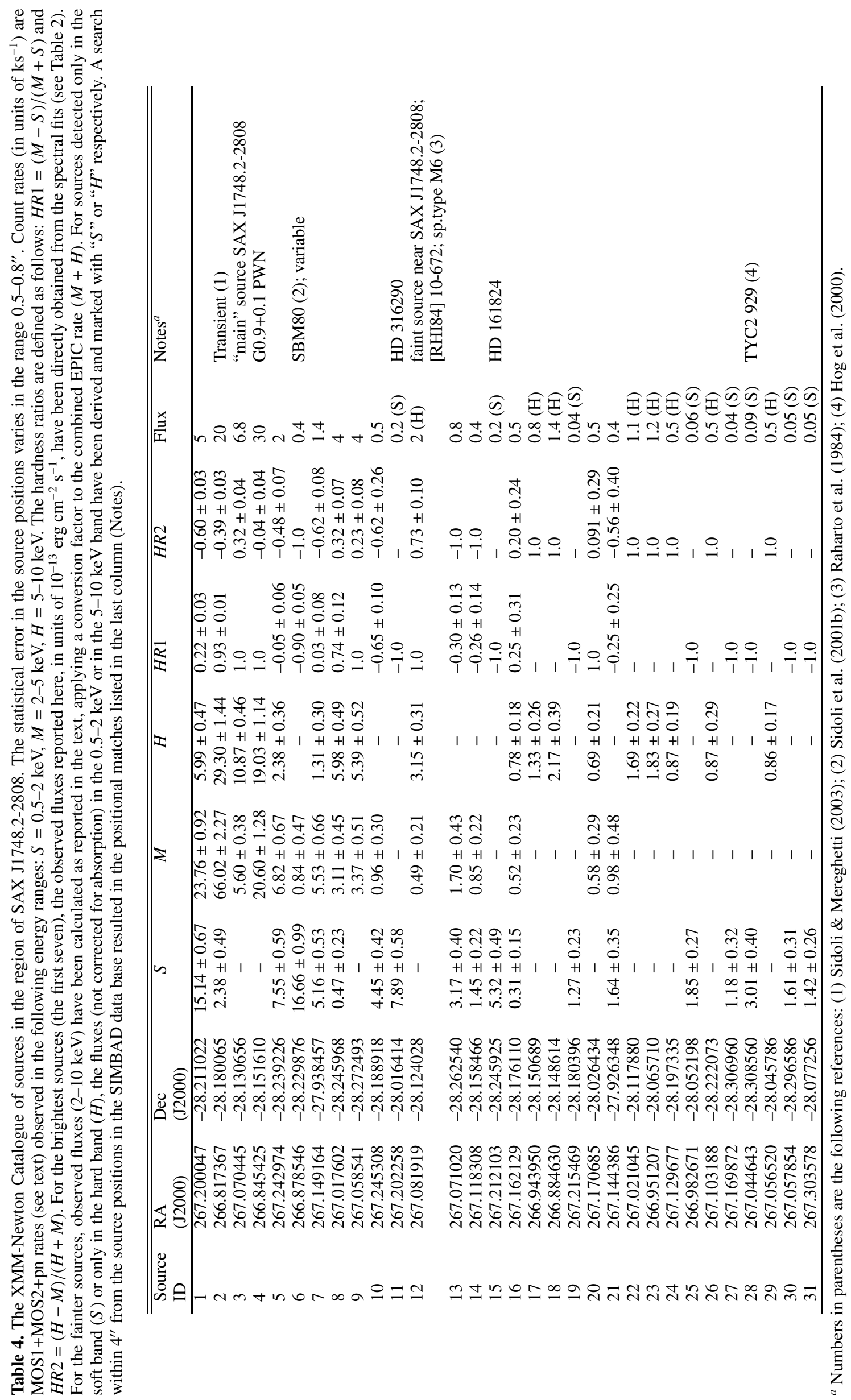


variability on timescales of years, while X-ray temporal variability and presence of radio emission are typical properties of AGNs.

The X-ray spectral properties of SAX J1748.2-2808 are reminiscent of the soft gamma-ray sources discovered with the INTEGRAL satellite (see e.g., Kuulkers 2005, for a review). Several of their X-ray counterparts display hard and heavily absorbed spectra, together with intense fluorescent Fe line emission, indicative of dense gaseous envelopes around the compact object, illuminated by the central source. In few of them, the association with $\mathrm{OB}$ optical counterparts and the detection of X-ray pulsations, suggest that they are highly absorbed HMXRBs, not detected in previous surveys at soft X-rays. The derived luminosity of these INTEGRAL sources is around $10^{36} \mathrm{erg} \mathrm{s}^{-1}$, although there is a large uncertainty in the distance estimates, and the true luminosity could be much less than this. SAX J1748.2-2808 lies in the direction of SgrD molecular cloud, near to SgrB2, which is an important site of star formation, so it is not unlikely that SAX J1748.2-2808 is indeed a HMXRB. The low X-ray luminosity suggests that it belongs to a class of massive X-ray binaries with low persistent emission (in the range $10^{34}-10^{35} \mathrm{erg} \mathrm{s}^{-1}$ ), wind-accreting and with no outbursts (e.g., 4U 2206+54, Masetti et al. 2004). On the other hand, these sources typically show temporal variability on different timescales (sometimes with flares), which has not been observed in SAX J1748.2-2808 (perhaps because of the poor coverage). However, wind-fed HMXRBs are usually quite stable X-ray emitters on long timescales (months or years). Low luminosity wind-accreting neutron stars has been predicted by Pfahl et al. (2002), who proposed that most of the faint sources detected in the Chandra survey of the GC (Wang et al. 2002) could be of this kind. A search for hard unidentified sources from ROSAT PSPC observations seems to confirm that a new class of fainter wind-fed X-ray binaries exists in our Galaxy (Suchkov \& Hanisch 2004).

Other kinds of galactic X-ray binaries, containing neutron stars or black-holes, seem to be unlikely; the luminosity $\left(\sim 10^{34} \mathrm{erg} \mathrm{s}^{-1}\right)$ suggests an object in quiescence: but low-mass $\mathrm{X}$-ray binaries (LMXRBs) in quiescence (soft X-ray transients) typically have much softer spectra (blackbody temperatures $\sim 0.1-0.3 \mathrm{keV}$; e.g., Verbunt \& Lewin 2005), while black-hole $\mathrm{X}$-ray novae in quiescence have much lower luminosities (Kong et al. 2002).

In conclusion, among the different hypotheses discussed above, the spectral shape (hard, non-thermal), X-ray luminosity the presence of Fe line emission, seem to favor a low luminosity HMXRB.
Acknowledgements. Based on observations obtained with XMM-Newton, an ESA science mission with instruments and contributions directly funded by ESA member states and the USA (NASA). We thank Giovanna Giardino, Nicola La Palombara and Silvano Molendi for useful discussions. This research has made use of the SIMBAD database, operated at CDS, Strasbourg, France. This publication makes use of data products from the Two Micron All Sky Survey, which is a joint project of the University of Massachusetts and the Infrared Processing and Analysis Center/California Institute of Technology, funded by the National Aeronautics and Space Administration and the National Science Foundation. The XMM-Newton data analysis is supported by the Italian Space Agency (ASI), through contract ASI/INAF I/023/05/0.

\section{References}

Baldi, A., Molendi, S., Comastri, A., et al. 2002, ApJ, 564, 190B

Cutri, R. M., Skrutskie, M. F., Van Dyk, S., et al. 2003, University of Massachusetts and Infrared Processing and Analysis Center (IPAC/California Institute of Technology)

Ebisawa, K., Maeda, Y., Kaneda, H., et al. 2001, Science, 293, 1633

Hog, E., Fabricius, C., Makarov, V. V., et al. 2000, A\&A, 355, L27

Jansen, F., Lumb, D., Altieri, B., et al. 2001, A\&A, 365, L1

Koyama, K., Awaki, H., Kunieda, H., et al. 1989, Nature, 339, 60

Kong, A. K. H., McClintock, J. E., Garcia, M. R., et al. 2002, ApJ, 570, 277

Kuulkers, E., in Interacting Binaries: Accretion, Evolution and Outcomes, ed. L. A. Antonelli, et al., Proc. of the Interacting Binaries Meeting of Cefalu, Italy, July 2004 (AIP), in press [arXiv: astro-ph/0504625]

Lis, D. C., \& Goldsmith, P. F. 1991, ApJ, 286, 232

Maccacaro, T., Gioia, M. I., Wolter, A., et al. 1988, ApJ, 326, 680

Masetti, N., Dal Fiume, D., Amati, L., et al. 2004, A\&A, 423, 311

Mereghetti, S., Sidoli, L., \& Israel, G. L. 1998, A\&A, 331, L77

Pfahl, E., Rappaport, S., \& Podsiadlowski, Ph. 2002, ApJ, 571, L37

Porquet, D., Decourchelle, A., \& Warwick, R. S. 2003, A\&A, 401, 197

Raharto, M., Hamajima, K., Ichikawa, T., et al. 1984, Ann. Tokyo Astron. Obs., 19,469

Revnivtsev, M. G., Churazov, E. M., Sazonov, S. Yu., et al. 2004, A\&A, 425, L49

Sidoli, L. 2000, Ph.D. Thesis, Univ. of Milan, Italy

Sidoli, L., Mereghetti, S., Treves, A., et al. 2001a, A\&A, 372, 651

Sidoli, L., Belloni, T., \& Mereghetti, S. 2001b, A\&A, 368, 835

Sidoli, L., \& Mereghetti, S. 2003, ATel, 147

Sidoli, L., Bocchino, F., Mereghetti, S., et al. 2004, MmSAI, 75, 507

Strüder, L., Briel, U., Dennerl, K., et al. 2001, A\&A, 365, L18

Suchkov, A. A., \& Hanish, R. J. 2004, ApJ, 612, 437

Terashima, Y., Iyomoto, N., Ho, L. C., et al. 2002, ApJS, 139, 1

Turner, M. J. L., Abbey, A., Arnaud, M., et al. 2001, A\&A, 365, L27

Verbunt, F., \& Lewin, W. H. G. 2005, to appear in Compact Stellar X-ray Sources, ed. W. H. G. Lewin, \& M. van der Klis (Cambridge University Press) [arXiv: astro-ph/0404136]

Walter, R., Courvoisier, T. J.-L., Foschini, L., et al. 2004, Proc. of the V INTEGRAL Workshop, 16-20 February 2004, Munich, Germany, ed. V. Schoenfelder, G. Lichti, \& C. Winkler (Noordwijk: ESA Publication Division), ESA SP-552, 417

Wang, Q. D., Gotthelf, E. V., \& Lang, C. C. 2002, Nature, 415, 148 